\title{
Workspace Analysis of a 4 Cable-Driven Spatial Parallel Robot
}

\author{
Atal Anil Kumar ${ }^{1}$, Jean-François Antoine ${ }^{1}$, Patrick Zattarin ${ }^{1}$, and Gabriel Abba ${ }^{1}$ \\ ${ }^{1}$ Arts et Métiers ParisTech, Université de Lorraine, LCFC, F-57000 Metz, France
}

\begin{abstract}
This paper presents the static equilibrium workspace of an under-constrained cable-driven robot with four cables taking into account the forces and the moments due to the forces acting on the moving platform. The problem is formulated as a non-linear optimization problem with maintaining static equilibrium as the objective function. The simulations are done using MATLAB. The maximum force on the cables and tilting angle of the platform are used to define the feasible static equilibrium workspace and the results obtained are used to finalize the design of the collaborative cable-driven robot to be installed in existing production lines for the agile handling of parts in a manufacturing industry.
\end{abstract}

Keywords: Static equilibrium workspace, under-constrained cable robot, collaborative cable-driven robot.

\section{Introduction}

A new class of parallel robots emerged in the late 1980s and early 1990s from the works of Landsberger (1985), Higuchi et al. (1988) and Albus et al. (1993) with a major modification in the structure of the traditional parallel robot. This class of manipulators is commonly referred to as the Cable-Driven Parallel Robots (CDPRs) in which a platform is driven by a set of cables whose length is adjusted by a winch consisting of a tensioning motor and spool or a linear actuator moving a pulley system (Merlet and Daney, 2010). Coordinated retraction and extension of cable control the position and orientation of the platform.

CDPRs possesses a number of desirable features, such as simple structure, large load capacity, high payload-to-weight ratio, and low inertia, compared to serial-link and other parallel type robots. Moreover, its simple design makes CDPRs low cost, portable, and reconfigurable (Verhoeven, 2004). CDPRs are well suited for a variety of applications (Gosselin, 2014) such as large-scale material handling and manufacturing process, building construction, rescue operations, aerial cameras, high-speed assembly and pick and place operations, large-scale radio telescopes, medical rehabilitation of people.

\footnotetext{
${ }^{1}$ We would like to thank the Robotix Academy for funding this work as a part of the project funded by INTERREG V-A Grand Region program.
} 
One of the earliest classifications of CDPRs was proposed by Cone (1985) and has been extended by Verhoeven (2004). A cable robot with m-cables and ndegrees of freedom is said to be completely restrained or fully-constrained if it has one cable more than the number of degrees of freedom, i.e. $m=n+1$. In such a type of arrangement, all degrees of freedom can be controlled through the cables. An incompletely restrained or under-constrained cable robot is one in which the number of cables is less than or equal to the number of degrees of freedom, i.e. $m$ $\leq n$. Such systems have one feasible solution for cable tensions and mostly rely on gravity for keeping the cables taut.

Though under-constrained CDPRs have received little attention in the literature (Fattah and Agrawal, 2006, Jiang and Kumar, 2010, Merlet, 2013), it is favorable to use CDPRs with a limited number of cables in several applications in which the task to be performed requires a limited number of controlled freedoms or a limitation of dexterity is acceptable in order to decrease complexity, cost, setup time, likelihood of cable interference etc. (Abbasnejad and Carricato, 2015). For example, CALOWI, a 4 cable-driven parallel robot designed at the LARM laboratory was intended to be used in a hospital environment (Ottaviano, 2007). Some important results on the stability analysis and kinematics of underconstrained parallel robots have been discussed by Merlet and Daney (2009), Carricato and Merlet (2013).

The major objective of the project is to design a collaborative cable-driven parallel robot for agile handling of parts in the manufacturing industry. Collaborative robots are a recent trend to allow the interaction of the workers and robot in a safe way making use of the worker's experience. The CDPR is planned to be installed in the existing lines of the industry where available space is reduced by already installed machines. In such a scenario, there is a severe space constraint to install new serial robots and would cost a lot to the company. Installing the robots on the ceiling would require reinforcement of the structure, again making the task costlier. Hence, a cable driven parallel robot with 4 cables is proposed as a solution. The number of cables is restricted to 4 for the ease of installation with simple anchor points in the environment and also to reduce the complexity of cable collision with the equipment installed on the shop floor. It is desired to have the actuators on the moving platform, unlike other designs in which the motors are placed in the base or fixed structure.

This paper presents the preliminary studies done to calculate the static equilibrium workspace of a 4 cable-driven parallel robot. Earlier works with such CDPRs take into account only the forces acting on the moving platform (pointmass). However, this work takes into account both the forces and the moments due to the forces acting on the moving platform with a given dimension. The results obtained from this study indicate that it is possible to achieve satisfactory results with 4 cables and the values of tensions obtained can be used in finalizing the parameters for the design of the CDPR. 
The paper is organized as follows: section 2 presents the kinematic and geometric modelling equations used in the study of CDPRs followed by section 3 which gives a brief about the workspace to be studied and non-linear solver used for solving the tension equation. The results are presented in section 4 followed by the conclusion and future work in section 5 .

\section{Kinematic and Geometric Modelling}

The equations used in the modeling of CDPR is presented in this section. The modeling and analysis methods developed for conventional rigid link manipulators cannot be directly applied to the cable-driven robots because of the unilateral constraints where the tensions in the cables must be considered (Gosselin, 2014). A general cable-driven parallel robot with $m$-cables is shown in figure1.

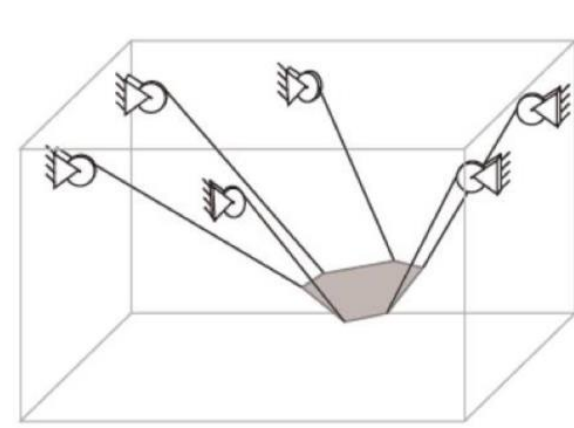

Figure 1. A simple arrangement of a CDPR

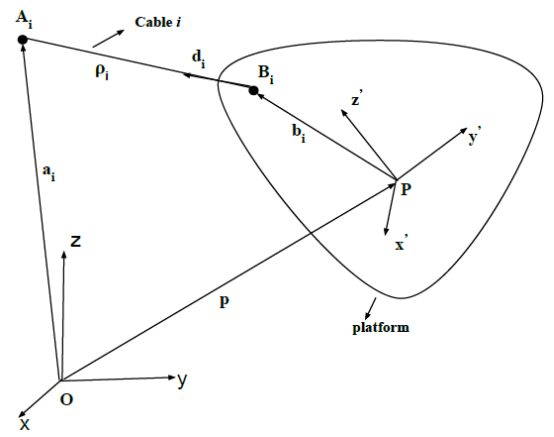

Figure 2. Kinematic model of a CDPR.

As seen in the figure above, a fixed reference frame $(O, x, y, z)$ is attached to the base of a CDPR and is referred to as the base frame. A moving reference frame $\left(P, x^{\prime}, y^{\prime}, z^{\prime}\right)$ is attached to the mobile platform where $P$ is the reference point of the platform to be positioned by the mechanism. The rotation matrix $Q$ gives the orientation of the moving frame with respect to the base frame.

From the figure 2, $a_{i}$ and $b_{i}$ are respectively defined as the vector connecting point $O$ to point $A_{i}$ and the vector connecting point $P$ of the platform to the point $B_{i}$, both vectors being expressed in the base frame. The position $p$ of the mobile platform is given by $\overrightarrow{O P}$.

Few assumptions are made in the modeling procedure to reduce the complexity of computation (Gosselin, 2014)

1) The mass of the cables is negligible and the cables are non-elastic.

2) The $i^{\text {th }}$ cable is assumed to be taut between points and is therefore considered a straight segment and is denoted by $\rho_{i}$. 
3) The moving platform is assumed to be a rigid body, defined by its mass and inertia matrix.

To identify the kinematic relationship between the cable space and body space for a CDPR, the loop closure equations are first developed for each chain of the CDPR.

The length of the cable can be calculated by using the vector loop equation given by

$$
\overrightarrow{d_{l}}=\overrightarrow{a_{\imath}}-\overrightarrow{b_{l}}-\vec{p}
$$

where, $\overrightarrow{\mathrm{d}_{1}}$ is the vector along cable $i$ pointing from $B_{i}$ to $A_{i}$ and $(i=1,2 \ldots m)$.

The length of the cable is calculated using

$$
\rho_{i}=\left\|\overrightarrow{d_{l}}\right\|^{2}
$$

Also using the above equation, the unit vector can be calculated by

$$
\widehat{d_{l}}=\left(\overrightarrow{a_{l}}-\overrightarrow{b_{l}}-\vec{p}\right) / \rho_{i}
$$

This basic model has been established in the early works on CDPRs and is purely based on the geometric analysis. This equation, however, does not take into account the ability of the mechanism to maintain the tensions in the cables. In order to address this issue, the concept of wrench matrix was introduced where statics of CDPR is considered.

When a tension $t_{i}$ is applied, the cable $i$ exerts at point $B_{i}$, a pure force $t_{i} \widehat{d}_{l}$ on the mobile platform. This pure force generates a moment $\overrightarrow{b_{l}} \times t_{i} \widehat{d}_{l}$ at the reference point $P$ of the mobile platform and the wrench (force/moment pair) applied at $P$ by the $i^{t h}$ cable is $t_{i} w_{i}$ with the wrench $w_{i}$ defined as

$$
w_{i}=\left[\begin{array}{c}
\hat{d}_{i} \\
\overrightarrow{b_{l}} \times \hat{d}_{i}
\end{array}\right]
$$

If $w_{p}$ is the total wrench applied at point $P$ by the $m$-cables then the relationship between the tensions in the cables and the wrench is written in matrix form as

$$
W \vec{t}=w_{p}
$$

where, $\overrightarrow{\mathrm{t}}$ is the vector of cable tensions and $W$ is the $6 \times m$ pose dependent wrench matrix.

Most of the analytical results proposed in the literature are based on this equation. By properly defining the vector $w_{p}$, different solutions can be obtained to understand the workspace of CDPRs. 


\section{Workspace Analysis}

Because of the unilateral constraint on the cables, the workspace analysis cannot be based solely on the change in cable lengths but must also take into account the statics of the manipulator (Gosselin, 2014).

The workspace of a CDPR is the set of position and orientation in which (Tavolieri et al., 2008) 1) The end-effector is controllable, 2) The tensions in the cables are positive, 3 ) The force values lie between a minimum and a maximum in order to avoid the cables break or become slack, 4) The end-effector is far from singularities, and 5) Cable wrapping is avoided.

Based on the conditions given above several different types of workspaces have been addressed in the literature, including (a) static equilibrium workspace (Pusey et al., 2004), (b) wrench closure workspace (WCW) (Gouttefarde et al., 2006), (c) wrench feasible workspace (WFW) (Ruiz et al., 2014), (d) dynamic workspace (Gagliardini et al., 2016), and (e) collision-free workspace (Ruiz et al., 2014).

The current work presents the static equilibrium workspace of the cable robot to be designed. The static equilibrium workspace (SEW) is the set of postures (position and orientation of the end-effector) that the end-effector can attain statically (only taking gravity into account). SEW is a subset of workplace for cable robots as all postures of the cable-driven robot are not statically attainable. It is not possible to calculate the WCW and WFW for the robot considered as they require the robot to be fully constrained or over-constrained. Hence it is important to have a good knowledge about SEW as it will help in the appropriate determination of the design parameters (motor speed, platform dimensions etc.).

In order to calculate the SEW of the CDPR, the static equilibrium condition of the cable robot is used to find the force on each cable. In other words, the equations given below which indicate the sum of forces $\left(\sum F_{i}, i=x, y, z\right)$ acting on the moving platform along the $\mathrm{x}, \mathrm{y}, \mathrm{z}$ direction and the sum of moments $\left(\left(\sum M_{i}, i=x, y, z\right)\right.$ acting on the moving platform along the $\mathrm{x}, \mathrm{y}, \mathrm{z}$ direction respectively, needs to be satisfied to achieve static equilibrium

$$
\sum F_{x}=0, \sum F_{y}=0, \sum F_{z}=0, \sum M_{x}=0, \sum M_{y}=0, \sum M_{z}=0
$$

The only external force acting on the platform is the gravitational force (along with the mass of the platform and payload acting at the center of mass of the moving platform) and all other external forces and moments will not be discussed in this paper. The equations described above are formulated as a nonlinear optimization problem and is solved using fsolve in MATLAB with static equilibrium condition as the objective function. 


\section{Results and Discussion}

The results of the simulation carried out in MATLAB are presented in this section. The SEW of the CDPR inside a room of dimension $5 \mathrm{~m} * 5 \mathrm{~m} * 3 \mathrm{~m}$ with a moving platform of size $0.5 \mathrm{~m} * 0.5 \mathrm{~m}$ and a total payload of $30 \mathrm{~kg}$ is shown in figure 3 . The constraints on the angle of inclination of the moving platform about $x$-axis and $y$-axis are $\pm 30^{\circ}$. The maximum and minimum allowed tensions in the cables were $500 \mathrm{~N}$ and $1 \mathrm{~N}$ respectively.

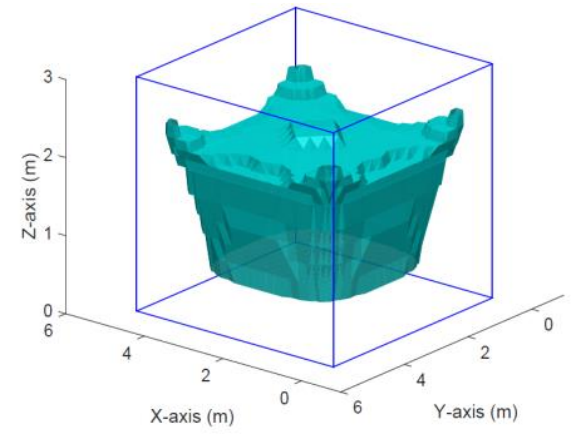

(a)

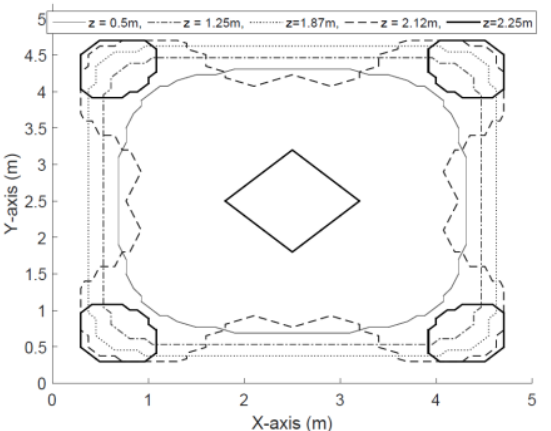

(b)

Figure 3. SEW of the CDPR for a payload of $30 \mathrm{~kg}$ (10kg platform+ 20kg payload) acting on the platform (a) 3-D representation and (b) 2-D representation.

The center of mass (CoM) of the moving platform was considered to be at a height of $0.2 \mathrm{~m}$ below the cable attachment points while the CoM of the payload was considered at a height of $0.4 \mathrm{~m}$ below the CoM of the platform. The step size was fixed along $\mathrm{x}$ and $\mathrm{y}$ axis to 0.05 while it was kept at 0.1 along the $\mathrm{z}$-axis. The search for possible workspace value was fixed to the region of $(0.3<x<4.7$, $0.3<y<4.7,0.5<z<2.3)$. The points were checked to see if they can be included in the SEW and it was found that $77 \%$ of the points from the selected region were satisfying the constraints considered during the simulation.

The dimensions of the platform were varied and the corresponding percentage of SEW is presented in the table given below. Since the final design includes the actuating motors on the moving platform, a dimension of $0.5 \mathrm{~m} * 0.5 \mathrm{~m}$ is considered to be a good compromise.

Table 1. $\%$ of SEW for platform with different dimensions

\begin{tabular}{|c|c|c|c|c|}
\hline Platform dimensions $(\mathrm{m})$ & $0.5^{*} 0.5$ & $0.75^{*} 0.75$ & $0.6^{*} 0.4$ & $0.8^{*} 0.6$ \\
\hline SEW \% & 68 & 56 & 68 & 58 \\
\hline
\end{tabular}

Since a 4 cable-driven spatial parallel robot has 4 degrees of freedom (the position $\mathrm{x}, \mathrm{y}, \mathrm{z}$ and one angle) that can be controlled by the cables, it is important 
to have an idea about how the platform orientation behaves as the simulation is done for static equilibrium condition.

Figures below show the variation of cable tensions and the corresponding changes in the value of the angles about $\mathrm{x}$-axis and $\mathrm{y}$-axis when the moving platform is moving in a plane at the center of the room $(\mathrm{z}=1.5 \mathrm{~m})$ considered. It can be seen that the change in angles about $\mathrm{x}$-axis and $\mathrm{y}$-axis is symmetrical under the consideration that the moving platform has equal length and width. The plot of cable tensions also exhibits symmetrical behavior with respect to $\mathrm{x}$ and $\mathrm{y}$ axis.

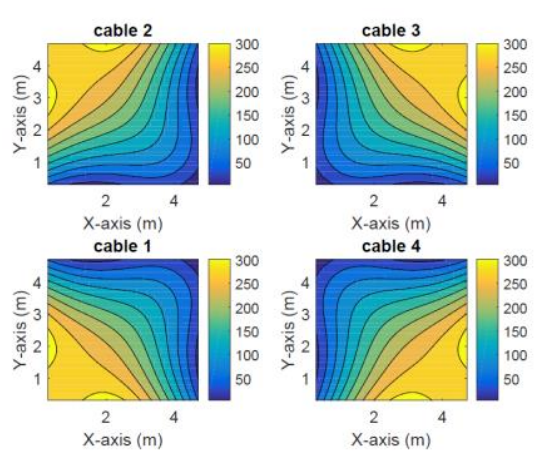

Figure 4. Tension distribution $(\mathrm{N})$ in 4 cables for $\mathrm{z}=1.5 \mathrm{~m}$.

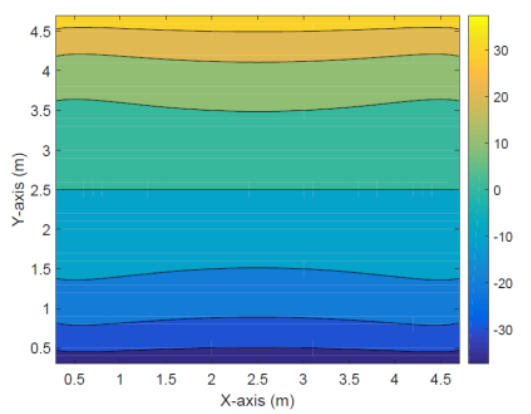

Figure 6. Variation of angle (in degrees) about $\mathrm{x}$-axis for $\mathrm{z}=1.5 \mathrm{~m}$.

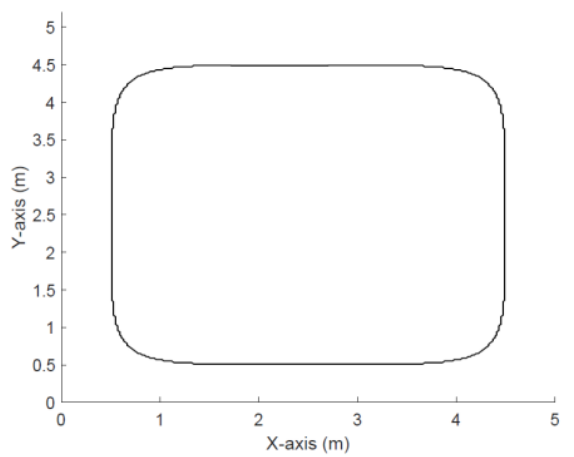

Figure 5. SEW for $\mathrm{z}=1.5 \mathrm{~m}$.

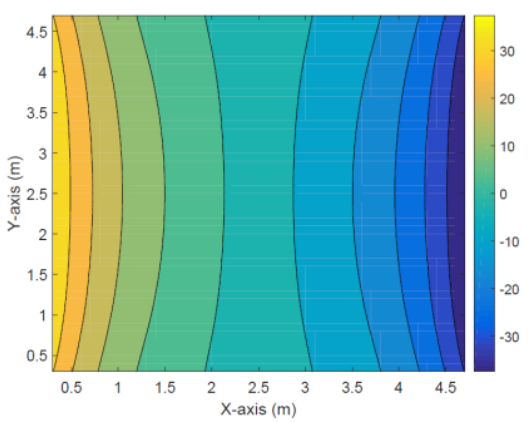

Figure 7. Variation of angle (in degrees) about $\mathrm{y}$-axis for $\mathrm{z}=1.5 \mathrm{~m}$.

\section{Conclusion}

The work presents the preliminary studies carried out in the design of a 4 cabledriven parallel robot. The static equilibrium workspace of the robot is calculated and the corresponding tensions for the cables are presented. It can be seen that it 
is possible to have a sufficiently large workspace for such robots by limiting the orientation of the platform within a certain range. Future work will include the calculation of dynamic feasible workspace taking into account the platform accelerations along with the performance evaluation of the workspace. The end product of the current work is to have a prototype of the robot considered, in the agile handling of parts in a manufacturing environment.

\section{Bibliography}

Albus, J., Bostelman, R., \& Dagalakis, N. (1993). The NIST robocrane. Journal of Field Robotics, 10(5). 709-724.

Cone, L. L. (1985). Skycam-an aerial robotic camera system. Byte, 10(10), 122.

Fattah A. and Agrawal S.K., (2006), On the design of cable-suspended planar parallel robots, ASME Journal of Mechanical Design,vol. 127, no. 5.1021-1028.

Gagliardini, L., Gouttefarde, M., \& Caro, S. (2018). Determination of a dynamic feasible workspace for cable-driven parallel robots. In Advances in Robot Kinematics 2016.Springer, Cham. 361-370.

Gosselin, C. (2014). Cable-driven parallel mechanisms: state of the art and perspectives. Mechanical Engineering Reviews, 1(1).

Gouttefarde, M., Merlet, J. P., \& Daney, D. (2006). Determination of the wrench-closure workspace of 6-DOF parallel cable-driven mechanisms. In Advances in Robot Kinematics. Springer, Dordrecht. 315-322.

Higuchi, T., Ming, A., \& Jiang-Yu, J. (1988, June). Application of multi-dimensional wire crane in construction. In 5th Int. Symp. on Robotics in Construction. 661-668.

Jiang Q. and Kumar V., (2010), The direct kinematics of objects suspended from cables, in ASME 2010 Int. Design Engineering Technical Conferences, vol. 2, Montreal, Canada, August 15-18 2010.193-202.

Landsberger, S. E. (1985). A new design for parallel link manipulators. In Proceedings of the 1985 IEEE International Conference of Systems, Man and Cybernetics. 812-814.

Merlet J.P., (2013), Wire-driven parallel robot: Open issues, in Romansy 19-Robot Design, Dynamics and Control, V. Padois, P. Bidaud, and O. Khatib, Eds. Springer, 2013. 3-10.

Merlet, J. P., \& Daney, D. (2010, May). A portable, modular parallel wire crane for rescue operations. In Robotics and Automation (ICRA), 2010 IEEE Int. Conf. 2834-2839.

Ottaviano, E., Ceccarelli, M., Paone, A., \& Carbone, G. (2005, April). A low-cost easy operation 4-cable driven parallel manipulator. In Robotics and Automation, 2005. ICRA 2005. Proceedings of the 2005 IEEE International Conference.4008-4013.

Pusey, J., Fattah, A., Agrawal, S.K., \& Messina, E. (2004). Design and workspace analysis of a 6-6 cable-suspended parallel robot. Mechanism and machine theory, 39(7).761-778.

Ruiz, A. L. C., Caro, S., Cardou, P., \& Guay, F. (2015). Arachnis: Analysis of robots actuated by cables with handy and neat interface software. In Cable-Driven Parallel Robots. Springer International Publishing. 293-305.

Tavolieri C., Merlet J.P., Ceccarelli, M., (2008). A workspace analysis of a overconstrained cable-based parallel manipulator by using the interval analysis. In 3rd International symposium on Multibody Systems and Mechatronics.1-13

Verhoeven, R. (2004). Analysis of the workspace of tendon-based Stewart platforms (Doctoral dissertation, Universität Duisburg-Essen). 